\title{
Colorimetric sensor for phosphate ions detection using 2- mercaptoethanesulfonate modified silver nanoplates
}

\author{
Chanika Pinyorospathum ${ }^{1}$, Poomrat Rattanarat ${ }^{1}$, Weena Siangproh ${ }^{2}$, Orawon Chailapakul11,3 \\ ${ }^{1}$ Electrochemical and Optical Spectroscopy Center of Excellence (EOSCE), Department of Chemistry, \\ Faculty of Science, Chulalongkorn University, 254 Phayathai Road, Pathumwan, Bangkok 10330, \\ Thailand. \\ ${ }^{2}$ Department of Chemistry, Faculty of Science, Srinakharinwirot University, 114 Sukhumvit 23, \\ Wattana, Bangkok 10110, Thailand. \\ ${ }^{3}$ Center of Excellence on Petrochemical and Materials Technology, Chulalongkorn University, 254 \\ Phayathai Road, Pathumwan, Bangkok 10330, Thailand. \\ corawon@chula.ac.th
}

\begin{abstract}
:
A new, distinctive, and inexpensive colorimetric sensor for the detection of phosphate ions ( $\mathrm{Pi}$ ) performed on paper-based analytical device (PAD) based on anti-aggregation of 2mercaptoethanesulfonate (MS) modified silver nanoplates is proposed. An aggregation of MS-AgNPIs due to the electrostatic interaction between the negatively charged sulfonate group on MS-AgNPIs and europium ions $\left(\mathrm{Eu}^{3+}\right)$ causes color change. Whereas, the color of MS-AgNPIs remains unchanged in the presence of premixed $\mathrm{Eu}^{3+}$ with $\mathrm{Pi}$ because the higher binding affinity of $\mathrm{Eu}^{3+}$ has towards $\mathrm{Pi}$ leaving the AgNPls dispersed. An apparent color changing from dark violet to pink with the increasing amount of $\mathrm{Pi}$ can be observed by naked eyes on PAD and can be used for quantification by the measurement of color intensity using ImageJ software. The aggregation and anti-aggregation of MSAgNPIs were characterized by TEM and UV-visible spectroscopy to confirm the mechanism. This method can detect $\mathrm{Pi}$ in the range of $5-150 \mathrm{ppm}$ with a detection limit of $1.67 \mathrm{ppm}$ (3 SD/Slope) and a limit of quantification equals to $5.07 \mathrm{ppm}$ (10 SD/Slope). The optimum parameters including $\mathrm{pH}$, concentration of $\mathrm{MS}$ and $\mathrm{Eu}^{3+}$, reaction time, and effects of interferences were studied. This method can be applied with the detection of $\mathrm{Pi}$ in real samples collecting from soils and in agree with the standard method (UV-visible spectrophotometry).
\end{abstract}

Key words: phosphate ions, silver nanoplates, paper-based analytical device, colorimetric method.

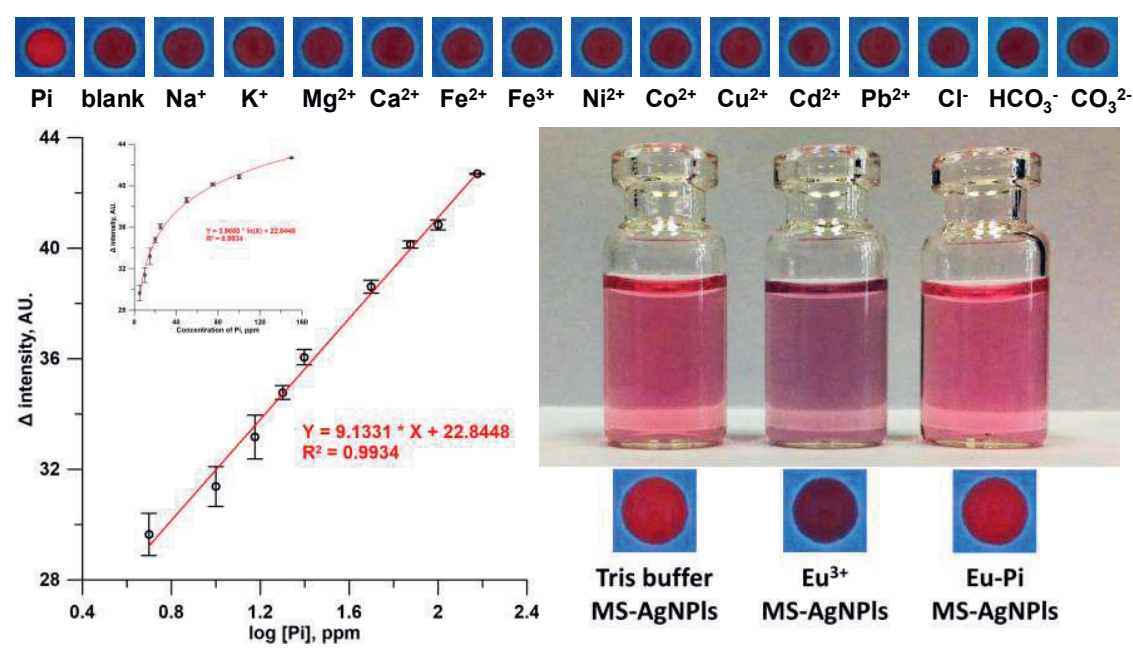

